\title{
Herschel/HIFI measurements of the ortho/para ratio in water towards Sagittarius B2(M) and W31C *
}

\author{
D. C. Lis ${ }^{1}$, T. G. Phillips ${ }^{1}$, P. F. Goldsmith ${ }^{13}$, D. A. Neufeld ${ }^{3}$, E. Herbst ${ }^{14}$, C. Comito ${ }^{8}$, P. Schilke ${ }^{8,12}$, H. S. P. Müller ${ }^{12}$, \\ E. A. Bergin ${ }^{2}$, M. Gerin ${ }^{9}$, T. A. Bell ${ }^{1}$, M. Emprechtinger ${ }^{1}$, J. H. Black ${ }^{23}$, G. A. Blake ${ }^{1}$, F. Boulanger ${ }^{24}$, E. Caux ${ }^{4,5}$, \\ C. Ceccarelli ${ }^{6}$, J. Cernicharo ${ }^{7}$, A. Coutens ${ }^{4.5}$, N. R. Crockett $^{2}$, F. Daniel ${ }^{7,9}$, E. Dartois ${ }^{24}$, M. De Luca ${ }^{9}$, \\ M.-L. Dubernet ${ }^{10,11}$, P. Encrenaz ${ }^{9}$, E. Falgarone ${ }^{9}$, T. R. Geballe ${ }^{26}$, B. Godard ${ }^{9}$, T. F. Giesen ${ }^{12}$, J. R. Goicoechea ${ }^{7}$, \\ C. Gry ${ }^{13}$, H. Gupta ${ }^{13}$, P. Hennebelle ${ }^{9}$, P. Hily-Blant ${ }^{6}$, R. Kołos ${ }^{27}$, J. Krełowski ${ }^{25}$, C. Joblin ${ }^{4,5}$, D. Johnstone ${ }^{15}$, \\ M. Kaźmierczak ${ }^{25}$, S. D. Lord $^{16}$, S. Maret ${ }^{6}$, P. G. Martin ${ }^{17}$, J. Martín-Pintado ${ }^{7}$, G. J. Melnick ${ }^{18}$, K. M. Menten ${ }^{8}$, \\ R. Monje ${ }^{1}$, B. Mookerjea ${ }^{28}$, P. Morris ${ }^{16}$, J. A. Murphy ${ }^{19}$, V. Ossenkopf ${ }^{12,20}$, J. C. Pearson ${ }^{13}$, M. Pérault ${ }^{9}$, C. Persson ${ }^{23}$, \\ R. Plume ${ }^{21}$, S.-L. Qin ${ }^{12}$, M. Salez , S. Schlemmer ${ }^{12}$, M. Schmidt ${ }^{29}$, P. Sonnentrucker ${ }^{3}$, J. Stutzki ${ }^{12}$, D. Teyssier ${ }^{30}$, \\ N. Trappe ${ }^{19}$, F. F. S. van der Tak $^{20}$, C. Vastel ${ }^{4,5}$, S. Wang ${ }^{2}$, H. W. Yorke ${ }^{13}$, S. Yu ${ }^{13}$, J. Zmuidzinas ${ }^{1}$, A. Boogert ${ }^{16}$, \\ N. Erickson ${ }^{22}$, A. Karpov ${ }^{1}$, J. Kooi ${ }^{1}$, F. W. Maiwald ${ }^{13}$, R. Schieder ${ }^{12}$, and P. Zaal ${ }^{20}$
}

(Affiliations are available on page 5 of the online edition)

Received 28 May 2010 / Accepted 18 June 2010

\section{ABSTRACT}

We present Herschel/HIFI observations of the fundamental rotational transitions of ortho- and para- $\mathrm{H}_{2}^{16} \mathrm{O}$ and $\mathrm{H}_{2}^{18} \mathrm{O}$ in absorption towards Sagittarius B2(M) and W31C. The ortho/para ratio in water in the foreground clouds on the line of sight towards these bright continuum sources is generally consistent with the statistical high-temperature ratio of 3 , within the observational uncertainties. However, somewhat unexpectedly, we derive a low ortho/para ratio of $2.35 \pm 0.35$, corresponding to a spin temperature of $\sim 27 \mathrm{~K}$, towards Sagittarius B2(M) at velocities of the expanding molecular ring. Water molecules in this region appear to have formed with, or relaxed to, an ortho/para ratio close to the value corresponding to the local temperature of the gas and dust.

Key words. astrochemistry - ISM: abundances - ISM: molecules - molecular processes - submillimeter: ISM

\section{Introduction}

Water molecules play an essential role in the physics and chemistry of the dense interstellar medium (ISM). Water is one of the main reservoirs of oxygen, and as an important coolant of dense gas it strongly affects its star formation properties. For a molecule with two hydrogen atoms, such as water, the ortho/para ratio is temperature dependent and, in principle, the temperature of the medium in which the proton spin state populations last equilibrated can be deduced from the observations. Water is an asymmetric top molecule, with energy levels labeled by the set of quantum numbers $J, K_{-1}, K_{+1}$, where $K_{-1}, K_{+1}$ are the limiting prolate and oblate symmetric top quantum numbers. Levels with $K_{-1}+K_{+1}$ even are para, and those with $K_{-1}+K_{+1}$ odd are ortho. There are no fast radiative transitions between the two species of water, ortho and para. However, a collision resulting in a proton exchange, with a proton or $\mathrm{H}_{3}^{+}$ion, can cause an ortho-para conversion in a water molecule. Such conversion can also result from interaction between a water molecule and the grain surface on which it is adsorbed. The lowest ortho state of water is $34.2 \mathrm{~K}$ above the para ground state. The ortho/para

* Herschel is an ESA space observatory with science instruments provided by European-led Principal Investigator consortia and with important participation from NASA. ratio in water is 3 in the high temperature limit. Departures from this limiting value are seen for nuclear spin temperatures below $\sim 50 \mathrm{~K}$, with the ratio dropping to 1.5 at $\sim 18 \mathrm{~K}$ (see Fig. 4 of Mumma et al. 1987).

The ground-state rotational transition of o-water at $557 \mathrm{GHz}$ was studied extensively in the ISM by the SWAS and Odin satellites (Melnick \& Bergin 2005; Hjalmarson 2004). However, the HIFI instrument (de Graauw 2010) aboard the Herschel Space Observatory (Pilbratt et al. 2010) allows for the first time heterodyne studies of the fundamental p-water line at $1113 \mathrm{GHz}$. Measurements of the ortho/para ratio in water, by means of absorption spectroscopy in cold foreground clouds on sightlines towards bright submillimeter continuum sources, can provide key insights into the thermal history of the gas. The ortho/para ratio in water has been measured in several solar system comets and the derived nuclear spin temperatures are typically of order $30 \mathrm{~K}$ (see Crovisier et al. 1997; Kawakita et al. 2004).

\section{Observations}

HIFI observations of the ortho and para $\mathrm{H}_{2}^{16} \mathrm{O}$ and $\mathrm{H}_{2}^{18} \mathrm{O}$ towards Sagittarius B2(M) and W31C presented here were carried out between 2010 March 1 and March 5, using the dual beam switch (DBS) observing mode, as part of guaranteed time 
key programs HEXOS: Herschel/HIFI observations of EXtraOrdinary Sources: The Orion and Sagittarius B2 star-forming regions and PRISMAS: PRobing InterStellar Molecules with Absorption line Studies. The source coordinates are: $\alpha_{J 2000}=$ $17^{\mathrm{h}} 47^{\mathrm{m}} 20.35^{\mathrm{s}}$ and $\delta_{J 2000}=-28^{\circ} 23^{\prime} 03.0^{\prime \prime}$ for Sagittarius B2(M) and $\alpha_{J 2000}=18^{\mathrm{h}} 10^{\mathrm{m}} 28.7^{\mathrm{s}}$ and $\delta_{J 2000}=-19^{\circ} 55^{\prime} 50.0^{\prime \prime}$ for W31C. The DBS reference beams lie approximately $3^{\prime}$ east and west (i.e. perpendicular to the roughly north-south elongation of Sagittarius B2). We used the HIFI wide band spectrometer (WBS) providing a spectral resolution of $1.1 \mathrm{MHz}\left(\sim 0.6 \mathrm{~km} \mathrm{~s}^{-1}\right.$ at $557 \mathrm{GHz}$ ) over a $4 \mathrm{GHz}$ IF bandwidth. The spectra presented here are equally-weighted averages of the $\mathrm{H}$ and $\mathrm{V}$ polarizations, reduced using HIPE (Ott 2010) with pipeline version 2.6. The resulting Level 2 double sideband (DSB) spectra were exported to the FITS format for a subsequent data reduction and analysis using the IRAM GILDAS package.

The band $1 \mathrm{a}, 1 \mathrm{~b}$ and $4 \mathrm{~b}$ spectral scans of Sagittarius B2(M) consist of DSB spectra with a redundancy of 8, which gives observations of a specific lower or upper sideband frequency with 8 different settings of the local oscillator (LO). This observing mode allows for the deconvolution and isolation of a single sideband (SSB) spectrum (Comito \& Schilke 2002). We applied the standard deconvolution routine within CLASS. The observations of water lines in W31C were obtained using the DBS single point observing mode with 3 shifted LO settings that were averaged to produce the final spectra. The HIFI beam size at 557 and $1113 \mathrm{GHz}$ is $38^{\prime \prime}$ and $21^{\prime \prime}$, respectively, with a main beam efficiency equal to $\sim 0.68$.

HIFI spectra towards strong continuum sources are affected by standing waves. For sources with narrow lines, these standing waves can be removed efficiently in the pipeline using the FitHifiFringe task in HIPE. However, in the case of Sagittarius B2(M), the period of standing waves is comparable to the line widths in the band 1 data. Given the high density of lines in this source in the low-frequency HIFI bands, the standing wave removal can lead to artifacts in the resulting spectra. We, therefore, have not attempted to "defringe" these data.

\section{Results}

Figure 1 shows spectra of the ground-state rotational transitions of o- and $\mathrm{p}-\mathrm{H}_{2}^{16} \mathrm{O}$ and $\mathrm{H}_{2}^{18} \mathrm{O}$ towards Sagittarius B2(M). Lines of $\mathrm{H}_{2}^{16} \mathrm{O}$ are completely saturated over a wide range of velocities. Absorption in the envelope of Sagittarius B2 is seen around $62 \mathrm{~km} \mathrm{~s}^{-1}$, while absorption at velocities between -140 and $40 \mathrm{~km} \mathrm{~s}^{-1}$ is due to the foreground gas.

The $\mathrm{o}-\mathrm{H}_{2}^{16} \mathrm{O}$ transition can be observed in HIFI bands $1 \mathrm{a}$ and 1b. While the two observations give the same line shape, the depth of the absorption is a few percent above the zero continuum level in band $1 \mathrm{a}$ and a few percent below the zero continuum level in band $1 \mathrm{~b}$ (Fig. 1, upper panel). This effect is due to small imbalances in the mixer sideband ratios. The sideband deconvolution can, in principle, fit the sideband gains. However, the $\mathrm{H}_{2} \mathrm{O}$ line is near the band edge and can only observed in the upper sideband in band 1a and only in the lower sideband in band $1 \mathrm{~b}$. The resulting determination of the sideband gains is thus not completely accurate. In the following analysis, we corrected for this instrumental effect by assuming that the deepest absorption in both $\mathrm{H}_{2}^{16} \mathrm{O}$ lines defines the zero continuum level.

Sagittarius B2(M) has a rich emission spectrum, which adds to the dust continuum, and against which the foreground clouds can absorb. Some of the strongest and most numerous emission lines seen in the spectrum are those of $\mathrm{SO}_{2}$, which we have

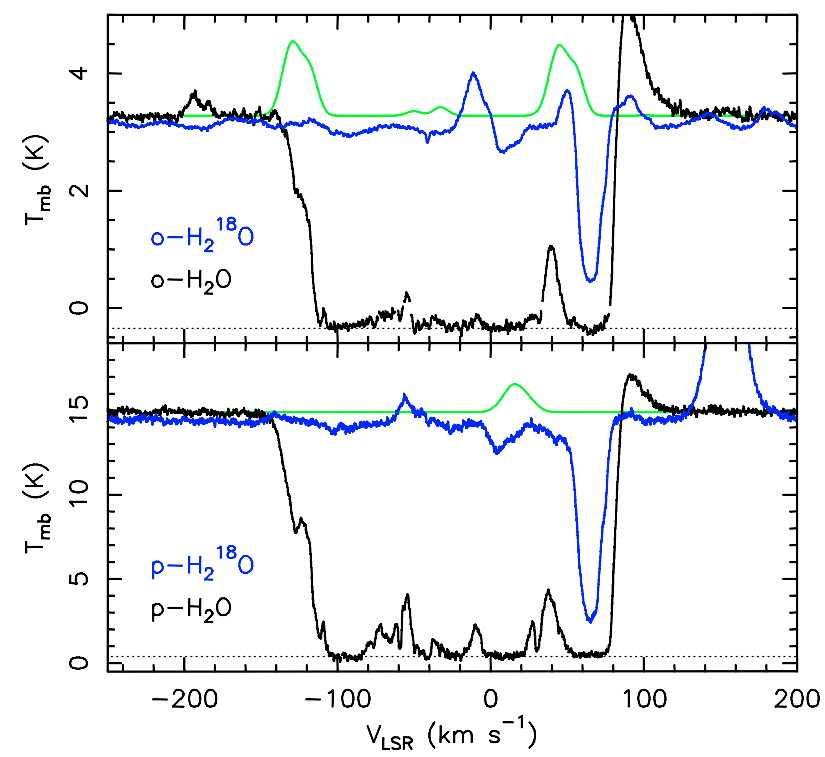

Fig. 1. (Upper) Spectra of the ground-state transitions of $\mathrm{o}-\mathrm{H}_{2}^{16} \mathrm{O}$ (band 1b) and $\mathrm{H}_{2}^{18} \mathrm{O}$ (band 1a) towards Sagittarius B2(M) (black and blue histograms, respectively). (Lower) Corresponding spectra of the $\mathrm{p}-\mathrm{H}_{2}^{16} \mathrm{O}$ and $\mathrm{H}_{2}^{18} \mathrm{O}$ transitions (band $4 \mathrm{~b}$ ). Green lines show the model for $\mathrm{SO}_{2}$ emission from the hot core described in the text.

modelled assuming LTE $^{1}$. The resulting model, shown as green lines in Fig. 1, includes two relatively strong $\mathrm{SO}_{2}$ lines within the $\mathrm{o}-\mathrm{H}_{2} \mathrm{O}$ spectrum, and a weaker line within the $\mathrm{p}-\mathrm{H}_{2} \mathrm{O}$ spectrum. These lines are included, as an additional background, in the calculation of the $\mathrm{H}_{2} \mathrm{O}$ optical depth. Another $\mathrm{SO}_{2}$ line is present in the $0-\mathrm{H}_{2}^{18} \mathrm{O}$ spectrum at $-15 \mathrm{~km} \mathrm{~s}^{-1}$ (Fig. 2, upper panel) and has been similarly modelled. The full source model of S.-L. Qin (private comm.) suggests that no other strong lines are present in our spectra at velocities corresponding to the foreground clouds.

Figure 2 (upper and middle panels) shows the o- and p- $\mathrm{H}_{2}^{16} \mathrm{O}$ and $\mathrm{H}_{2}^{18} \mathrm{O}$ spectra divided by the background emission, including dust continuum and the $\mathrm{SO}_{2}$ lines. The $\mathrm{o}-\mathrm{H}_{2}^{16} \mathrm{O}$ is an equallyweighted average of the band $1 \mathrm{a}$ and $1 \mathrm{~b}$ data. The $\mathrm{H}_{2}^{16} \mathrm{O}$ lines are saturated over a wide range of velocities and thus unusable for a quantitative analysis. However, we have identified several velocity ranges with moderate saturation levels, marked with thick horizontal lines in Fig. 2. These can be identified with the expanding molecular ring $\left(<-50 \mathrm{~km} \mathrm{~s}^{-1}\right)$, a transition between the $4 \mathrm{kpc}$ arm and the Orion arm $\left(-12\right.$ to $\left.-7 \mathrm{~km} \mathrm{~s}^{-1}\right)$, the Sagittarius $\operatorname{arm}\left(5\right.$ to $\left.20 \mathrm{~km} \mathrm{~s}^{-1}\right)$, and the Scutum arm (27 to $35 \mathrm{~km} \mathrm{~s}^{-1}$; possibly blended with the Sagittarius B2 envelope; see e.g. Neufeld et al. 2000). Assuming that the foreground absorption completely covers the continuum and all water molecules are in the ground state (a reasonable assumption for the diffuse foreground clouds given the very high spontaneous emission rate coefficients for the ground-state water lines, $3.458 \times 10^{-3}$ and $1.842 \times 10^{-2} \mathrm{~s}^{-1}$ for the ortho and para lines, respectively), we derive optical depths of the o- and p-water lines $\left(\tau=-\ln I / I_{o}\right)$. The resulting optical depth ratio is shown in Fig. 2 (lower panel; left intensity scale). An ortho/para optical depth ratio of 1 corresponds to a column density ratio of 2 . The resulting ortho/para

1 We made use of the myXCLASS program (http://www.astro. uni-koeln.de/projects/schilke/XCLASS), which accesses the CDMS (Müller et al. 2001, 2005; http://www.cdms.de) and JPL (Pickett et al. 1998; http://spec.jpl.nasa.gov) molecular databases. 
D. C. Lis et al.: Ortho/para ratio in interstellar water

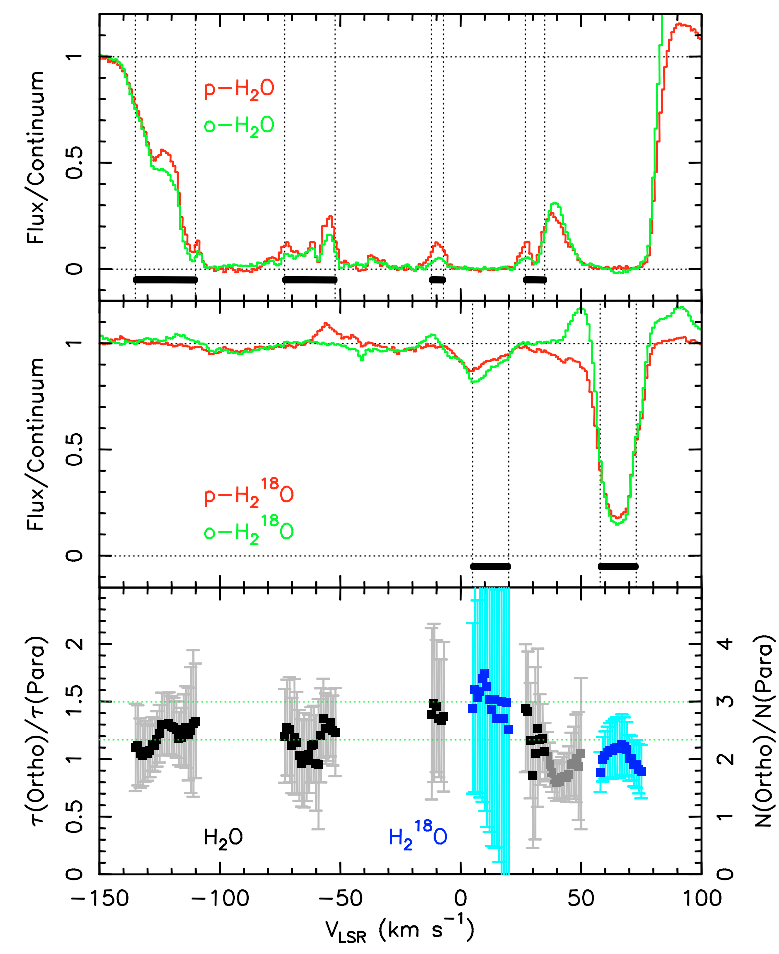

Fig. 2. (Upper) Spectra of the ground state o- and $\mathrm{p}-\mathrm{H}_{2}^{16} \mathrm{O}$ lines towards Sagittarius B2(M) normalized by the continuum (green and red histograms, respectively). (Middle) Corresponding spectra of the $\mathrm{H}_{2}^{18} \mathrm{O}$ lines. (Lower) The ortho/para optical depth ratio (left scale) and column density ratio (right scale) as a function of LSR velocity. Black and blue points correspond to the $\mathrm{H}_{2}^{16} \mathrm{O}$ and $\mathrm{H}_{2}^{18} \mathrm{O}$ measurements, respectively.

column density ratio is given by the right hand scale in Fig. 2 (lower panel).

The uncertainty in the line optical depth in a given velocity channel is given by $\delta \tau=\exp (\tau) \times \delta I / I_{o}$. The errorbars in Fig. 2 (lower panel), are computed under the conservative assumption $\delta I / I_{o}=0.05$ (maximum uncertainty, dominated by the standing waves, based on a comparison of the continuum normalized spectra of the o- $\mathrm{H}_{2} \mathrm{O}$ line in bands $1 \mathrm{a}$ and $1 \mathrm{~b}$; the signal-to-noise ratio for the $\mathrm{p}-\mathrm{H}_{2} \mathrm{O}$ line is comparable, given the stronger continuum, and the amplitude of the standing waves also scales with the continuum strength). Relative uncertainties of the o- and pwater optical depths have been added in quadrature.

Table 1 gives weighted averages of the ortho/para column density ratio in different velocity ranges towards Sagittarius B2(M), along with the corresponding uncertainties. Since the individual measurements are not independent but dominated by instrumental systematics, we use a more conservative estimate of the uncertainty of the mean for the two velocity intervals corresponding to the expanding molecular ring, computed from the peak-peak variation between the individual data points. The $\mathrm{H}_{2} \mathrm{O}$ (ortho+para) column densities are also included, assuming an $\mathrm{H}_{2}^{16} \mathrm{O} / \mathrm{H}_{2}^{18} \mathrm{O}$ ratio of 500 for the $5-20 \mathrm{~km} \mathrm{~s}^{-1}$ component. We compute $\mathrm{H}_{2}$ column densities in the foreground gas using the method employed in Lis et al. (2001), based on ${ }^{13} \mathrm{CO}$ absorption data, assuming a $\mathrm{CO}$ abundance of $1 \times 10^{-4}$ and a ${ }^{12} \mathrm{CO} /{ }^{13} \mathrm{CO}$ ratio of 60 in the local gas in the Sagittarius arm (5$20 \mathrm{~km} \mathrm{~s}^{-1}$ velocity range) and 30 in the remaining velocity intervals. The resulting column densities should be accurate to within a factor of 2. The $\mathrm{H}_{2} \mathrm{O}$ abundance in the various components is generally consistent with that derived by Neufeld et al. (2000; $4-7 \times 10^{-7}$ ). The derived $\mathrm{H}_{2} \mathrm{O}$ abundance in the $5-20 \mathrm{~km} \mathrm{~s}^{-1}$ component, based on the $\mathrm{H}_{2}^{18} \mathrm{O}$ measurements, is a factor of 3-6

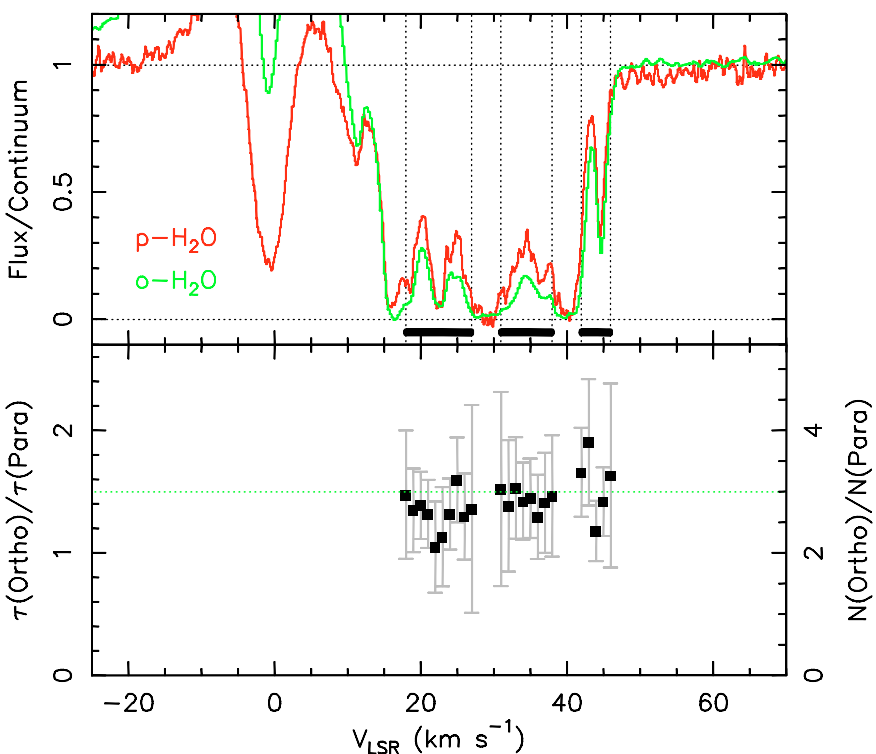

Fig. 3. (Upper) Spectra of the ground state o- and $\mathrm{p}-\mathrm{H}_{2}^{16} \mathrm{O}$ lines towards W31C normalized by the continuum (green and red histograms, respectively). (Lower) The ortho/para optical depth ratio (left scale) and column density ratio (right scale) as a function of LSR velocity.

Table 1. Column densities $\left(\mathrm{cm}^{-2}\right)$ and ortho/para ratios towards Sagittarius B2(M).

\begin{tabular}{ccccc}
\hline \hline$V\left(\mathrm{~km} \mathrm{~s}^{-1}\right)$ & $O / P$ & $N\left(\mathrm{H}_{2} \mathrm{O}\right)$ & $N\left(\mathrm{H}_{2}\right)$ & $X\left(\mathrm{H}_{2} \mathrm{O}\right)$ \\
\hline-135 to -110 & $2.35 \pm 0.3$ & $2.0 \times 10^{14}$ & $5 \times 10^{20}$ & $4 \times 10^{-7}$ \\
-73 to -52 & $2.35 \pm 0.4$ & $3.7 \times 10^{14}$ & $6 \times 10^{20}$ & $6 \times 10^{-7}$ \\
-12 to -7 & $2.8 \pm 0.5$ & $1.2 \times 10^{14}$ & $4 \times 10^{20}$ & $3 \times 10^{-7}$ \\
5 to 20 & $3.0 \pm 0.6$ & $6.7 \times 10^{15}$ & $3 \times 10^{21}$ & $2 \times 10^{-6}$ \\
27 to 35 & $2.3 \pm 0.3$ & $1.7 \times 10^{14}$ & $6 \times 10^{20}$ & $3 \times 10^{-7}$ \\
\hline
\end{tabular}

higher than that derived for the other components based on the $\mathrm{H}_{2}^{16} \mathrm{O}$ data. However, the $\mathrm{H}_{2}^{18} \mathrm{O}$ optical depth in this velocity range is low leading to large uncertainties. The water column density and abundance in this velocity range would be a factor of 2 lower if the gas responsible for the absorption is located in the Galactic center region $\left(\mathrm{H}_{2}^{16} \mathrm{O} / \mathrm{H}_{2}^{18} \mathrm{O}\right.$ ratio of 250$)$ rather than in the Sagittarius arms.

The ortho/para ratio in the two velocity ranges between -12 and $20 \mathrm{~km} \mathrm{~s}^{-1}$ is consistent with the high-temperature limit of 3 , within the uncertainties. The ratio in the Scutum arm, at 27 to $35 \mathrm{~km} \mathrm{~s}^{-1}$ is lower than 3 . However, this component may be blended with the Sagittarius B2 envelope. The low ortho/para ratios derived at the envelope velocities, from both $\mathrm{H}_{2}^{16} \mathrm{O}$ and $\mathrm{H}_{2}^{18} \mathrm{O}$ data, are likely caused by the excitation effects. Given the higher density of the gas, the assumption that all water molecules are in the ground state is no longer correct. The column density of $\mathrm{o}-\mathrm{H}_{2} \mathrm{O}$ is more strongly affected, resulting in a lower apparent ortho/para ratio. In fact, we do see wings of the $\mathrm{o}-\mathrm{H}_{2}^{18} \mathrm{O}$ line in emission at the envelope velocities (Fig. 2; middle panel). Deriving the ortho/para ratio in water in the Sagittarius B2 envelope requires detailed radiative transfer modelling and is beyond the scope of the present Letter.

We derive a low ortho/para ratio, $2.35 \pm 0.35$, at velocities $<-50 \mathrm{~km} \mathrm{~s}^{-1}$, corresponding to the expanding molecular ring. In this case, the measurement uncertainties are small enough that the ortho/para ratio of 3 appears to be ruled out by our data.

The o- and p-water spectra towards W31C, normalized by the continuum, are shown in Fig. 2 (upper panel). Weak p- $\mathrm{H}_{2}^{18} \mathrm{O}$ absorption is seen at the cloud systemic velocity, but no o- or 
$\mathrm{p}-\mathrm{H}_{2}^{18} \mathrm{O}$ absorption is detected in the foreground clouds. Once again, we have identified 3 velocity ranges where the o- and p$\mathrm{H}_{2}^{16} \mathrm{O}$ lines are not completely saturated (thick horizontal lines in Fig. 2, upper panel). The resulting ortho/para ratio is relatively uniform, $2.8 \pm 0.2$, consistent with the high-temperature ratio of 3 within the measurement uncertainty (Fig. 2, lower panel).

\section{Discussion}

In the high-temperature limit one might expect an ortho/para ratio close to 3 , when water is first formed. In the gas-phase, the excess energy of the exothermic reactions (e.g., recombination of $\mathrm{H}_{3} \mathrm{O}^{+}$) could lead to spin equilibration. For water molecules desorbed from ices on grain surfaces, one might also expect the initial ortho/para ratio to be 3-if there is enough energy to desorb a water molecule, either thermally or non-thermally, there is likely enough energy to populate numerous rotational states of the gas-phase species, both ortho and para, preserving a ratio close to 3 independent of the ortho/para ratio in the ice. However, the excess energy of formation is shared with the surface and the water ortho/para ratio may rapidly equilibrate at the grain temperature (e.g., Limbach et al. 2006).

Once water molecules are in the gas phase, collisions with atomic and molecular ions $\left(\mathrm{H}^{+}\right.$and $\left.\mathrm{H}_{3}^{+}\right)$can lead to proton exchanges and, over time, produce a gas-phase ortho/para ratio below 3 and potentially commensurate with the gas temperature (see the discussion of $\mathrm{H}_{2}$ conversion by Flower et al. 2006). As the temperature approaches zero, all water molecules will be in the ground para state if collisions with ions can efficiently exchange the ortho and para states. If there are no ions present, and therefore no barrierless collisions to exchange the ortho and para states of water, the ortho/para ratio of 3 will remain, independent of the temperature. Since ions are present in the ISM, we can expect the water ortho/para ratio to be generally lower than 3, given sufficient time. The time scale of the ortho/para conversion depends on the gas density. In dense clouds, there is a greater likelihood that the ortho/para ratio equilibrates at the gas kinetic temperature, as long as the density of ions is sufficient. In warmer, diffuse clouds, equilibrium would favor higher ortho/para ratios, but at lower densities equilibrium is less likely. Assuming a gas density of $10^{4} \mathrm{~cm}^{-3}$, a fractional abundance of protonated ions of $10^{-8}$, and a rate coefficient of $10^{-9} \mathrm{~cm}^{-3} \mathrm{~s}^{-1}$, we estimate the time scale of the ortho-para equilibration to be of order $3 \times 10^{5}$ years.

We derive a low ortho/para ratio of $2.35 \pm 0.35$, corresponding to a spin temperature of $\sim 27 \mathrm{~K}$, towards Sagittarius B2(M) at velocities corresponding to the expanding molecular ring. The low ortho/para ratio may suggest water formation on dust grains, with water molecule spin populations equilibrated at the dust temperature. Alternatively, the high-temperature ratio of 3 may have relaxed in the gas-phase to a value in line with the kinetic temperature of these clouds - a gas/dust temperature of order $30 \mathrm{~K}$ is quite reasonable for these relatively diffuse clouds within $\sim 200 \mathrm{pc}$ from the Galactic center (e.g., Tieftrunk et al. 1994). The water ortho/para ratio has now been measured in atmospheres of 9 solar system comets. The derived spin temperatures range between 23 and $34 \mathrm{~K}$, with an average of $\sim 29 \mathrm{~K}$ (Crovisier, priv. comm.), close to our measurement in the expanding molecular ring.

The higher ortho/para ratio, consistent with the statistical ratio of 3, that we derive towards Sagittarius B2(M) in the velocity range -12 to $20 \mathrm{~km} \mathrm{~s}^{-1}$ may indicate higher gas or dust temperatures (e.g., Gardner et al. 1988). Schilke et al. (2010) also derive a higher $\mathrm{H}_{2} \mathrm{O}^{+}$spin temperature towards this source at positive velocities, as compared to the line-of-sight clouds at negative velocities. In addition, strong chloronium absorption has been detected towards Sagittarius B2(S) at velocities between -10 and $20 \mathrm{~km} \mathrm{~s}^{-1}$ (Lis et al. 2010). Since the $\mathrm{H}_{2} \mathrm{Cl}^{+}$abundance is enhanced in warm, UV-irradiated regions, this indicates the presence of a warm, diffuse gas component in this velocity range.

In summary, in the diffuse regions studied here, both water formation mechanisms (gas and solid state) can contribute. With sufficient time (or higher densities with subsequently faster gasphase reactions) chemistry in the gas can drive the spin temperature towards the gas temperature. In addition, it has been postulated (Hollenbach et al. 2009) that photodesorption of water ices in low-density environments can release frozen molecules with a spin temperature that coincides with that of the dust grains. If the gas and dust temperatures are not equal this can lead to a ratio that reflects a mixture of these effects. Modeling the ortho/para ratio evolution is beyond the scope of this Letter, but the theme is that a ratio below 3 reflects cold environments (gas or dust temperatures below about $50 \mathrm{~K}$ ).

With improved calibration and better understanding of the instrumental effects, more accurate determination of the water ortho/para ratio in these and other sources will be possible in the future. However, the present work clearly demonstrates the outstanding spectroscopic capabilities of HIFI for providing robust constraints for the physical conditions and chemistry of the ISM.

Acknowledgements. HIFI has been designed and built by a consortium of institutes and university departments from across Europe, Canada and the United States under the leadership of SRON Netherlands Institute for Space Research, Groningen, The Netherlands and with major contributions from Germany, France and the US. Consortium members are: Canada: CSA, U.Waterloo; France: CESR, LAB, LERMA, IRAM; Germany: KOSMA, MPIfR, MPS; Ireland, NUI Maynooth; Italy: ASI, IFSI-INAF, Osservatorio Astrofisico di Arcetri-INAF; Netherlands: SRON, TUD; Poland: CAMK, CBK; Spain: Observatorio Astronomico Nacional (IGN), Centro de Astrobiología (CSIC-INTA). Sweden: Chalmers University of Technology-MC2, RSS \& GARD; Onsala Space Observatory; Swedish National Space Board, Stockholm University - Stockholm Observatory; Switzerland: ETH Zurich, FHNW; USA: Caltech, JPL, NHSC. Support for this work was provided by NASA through an award issued by JPL/Caltech. D. C. L. is supported by the NSF, award AST-0540882 to the CSO. A portion of this research was performed at the Jet Propulsion Laboratory, California Institute of Technology, under contract with the National Aeronautics and Space Administration.

\section{References}

Comito, C., \& Schilke, P. 2002, A\&A, 395, 357

Crovisier, J., Leech, K., Bockelée-Morvan, D., et al. 1997, Science, 275, 1904 de Graauw, Th., Helmich, F. P., Phillips, T. G., et al. 2010, A\&A, 518, L6

Flower, D., Pineau des Forêts, G., \& Walmsley, C. M. 2006, A\&A, 449, 621 Gardner, F. F., Boes, F., \& Winnewisser, G. 1988, A\&A, 196, 207

Greaves, J. S., \& Nyman, L.-A. 1996, A\&A, 305, 950

Hjalmarson, А̊ 2004, ASR, 34, 504

Hollenbach, D., Kaufman, et al. 2009, ApJ, 690, 1497

Kawakita, H., Watanabe, J., et al. 2004, ApJ, 601, 1152

Limbach, H.-H., Buntkowsky, G., et al. 2006, ChemPhysChem, 7, 551

Lis, D. C., Keene, J., Phillips, T. G., et al. 2001, ApJ, 561, 823

Lis, D. C., Pearson, J. C., Neufeld, D. A., et al. 2010, A\&A, 521, L9

Melnick, G. J., \& Bergin, E. A. 2005, ASR, 36, 1027

Müller, H. S. P., Thorwirth, S., et al. 2001, A\&A, 370, L49

Müller, H. S. P., Schlöder, et al. G. 2005, J. Mol. Struct., 742, 215

Mumma, M., Weaver, H. A., \& Larson, H. P. 1987, A\&A, 187, 419

Neufeld, D. A., Ashby, M. L. N., Bergin, E. A., et al. 2000, ApJ, 539, L111

Ott, S. 2010, in Astronomical Data Analysis Software and Systems XIX, ed. Y.

Mizumoto, K.-I. Morita, \& M. Ohishi, ASP Conf. Ser., in press

Pickett, H. M., Poynter, R. L., Cohen, E. A., et al. 1998, J. Quant. Spectrosc. Radiat. Transfer, 60, 883

Pilbratt, G. L., Riedinger, J. R., Passvogel, T., et al. 2010, A\&A, 518, L1

Schilke, P., Comito, C., Müller, H. S. P., et al. 2010, A\&A, 521, L11

Tieftrunk, A., Pineau des Forêts, G., et al. 1994, A\&A, 289, 579 
1 California Institute of Technology, Cahill Center for Astronomy and Astrophysics 301-17, Pasadena, CA 91125, USA

e-mail: dcl@caltech.edu

2 Department of Astronomy, University of Michigan, 500 Church Street, Ann Arbor, MI 48109, USA

3 Department of Physics and Astronomy, Johns Hopkins University, 3400 North Charles Street, Baltimore, MD 21218, USA

${ }^{4}$ Centre d'Étude Spatiale des Rayonnements, Université de Toulouse [UPS], 31062 Toulouse Cedex 9, France

5 CNRS/INSU, UMR 5187, 9 avenue du Colonel Roche, 31028 Toulouse Cedex 4, France

${ }^{6}$ Laboratoire d'Astrophysique de l'Observatoire de Grenoble, BP 53, 38041 Grenoble Cedex 9, France

7 Centro de Astrobiología (CSIC/INTA), Laboratiorio de Astrofísica Molecular, Ctra. de Torrejón a Ajalvir, km 4 28850, Torrejón de Ardoz, Madrid, Spain

8 Max-Planck-Institut für Radioastronomie, Auf dem Hügel 69, 53121 Bonn, Germany

9 LERMA, CNRS UMR8112, Observatoire de Paris and École Normale Supérieure, 24 rue Lhomond, 75231 Paris Cedex 05, France

${ }_{10}$ LPMAA, UMR7092, Université Pierre et Marie Curie, Paris, France

11 LUTH, UMR8102, Observatoire de Paris, Meudon, France

12 I. Physikalisches Institut, Universität zu Köln, Zülpicher Str. 77, 50937 Köln, Germany

13 Jet Propulsion Laboratory, Caltech, Pasadena, CA 91109, USA
14 Departments of Physics, Astronomy and Chemistry, Ohio State University, Columbus, OH 43210, USA

15 National Research Council Canada, Herzberg Institute of Astrophysics, 5071 West Saanich Road, Victoria, BC V9E 2E7, Canada

16 Infrared Processing and Analysis Center, California Institute of Technology, MS 100-22, Pasadena, CA 91125, USA

17 Canadian Institute for Theoretical Astrophysics, University of Toronto, 60 St George St, Toronto, ON M5S 3H8, Canada

18 Harvard-Smithsonian Center for Astrophysics, 60 Garden Street, Cambridge MA 02138, USA

19 National University of Ireland Maynooth. Ireland

20 SRON Netherlands Institute for Space Research, PO Box 800, 9700 $\mathrm{AV}$, Groningen, The Netherlands

21 Department of Physics and Astronomy, University of Calgary, 2500 University Drive NW, Calgary, AB T2N 1N4, Canada

22 University of Massachusetts, Astronomy Dept., 710 N. Pleasant St., LGRT-619E, Amherst, MA 01003-9305, USA

${ }^{23}$ Chalmers University of Technology, Göteborg, Sweden

${ }^{24}$ Institut d'Astrophysique Spatiale (IAS), Orsay, France

25 Nicolaus Copernicus University, Torun, Poland

${ }^{26}$ Gemini Telescope, Hilo, Hawaii, USA

27 Institute of Physical Chemistry, PAS, Warsaw, Poland

28 Tata Institute of Fundamental Research, Homi Bhabha Road, Mumbai 400005, India

29 Nicolaus Copernicus Astronomical Center, Poland

30 European Space Astronomy Centre, ESA, Madrid, Spain 